ÉGYPTE monde arabe

\section{Égypte/Monde arabe}

$3 \mid 2006$

Terrains d'Égypte, anthropologies contemporaines

\title{
«La vie du musicien est comme la vapeur d'eau, elle monte et disparaît »
}

(à propos de musiciens, de mariages et de citadins au Caire)

\section{Nicolas Puig}

\section{OpenEdition}

\section{Journals}

Édition électronique

URL : https://journals.openedition.org/ema/1077

DOI : 10.4000/ema.1077

ISSN : 2090-7273

Éditeur

CEDEJ - Centre d'études et de documentation économiques juridiques et sociales

Édition imprimée

Date de publication : 31 décembre 2006

Pagination : $35-60$

ISSN : 1110-5097

\section{Référence électronique}

Nicolas Puig, « « La vie du musicien est comme la vapeur d'eau, elle monte et disparaît » », Égypte/ Monde arabe [En ligne], 3 | 2006, mis en ligne le 08 juillet 2008, consulté le 07 juillet 2022. URL : http:// journals.openedition.org/ema/1077; DOI : https://doi.org/10.4000/ema.1077 


\title{
« LA VIE DU MUSICIEN EST COMME LA VAPEUR D’EAU, ELLE MONTE ET DISPARAÎT »
}

\author{
(À PROPOS DE MUSICIENS, DE MARIAGES ET DE CITADINS AU CAIRE)
}

$\int^{\prime}$

'ai retrouvé cette phrase sibylline au fond d'un carnet datant des débuts de I'enquête. Je la tiens d'un certain Abû Magdî et I'ai consignée lors d'une conversation dans l'un des cafés de l'avenue Muhammad Ali où se regroupent depuis près d'un siècle les musiciens spécialisés dans l'animation des fêtes populaires. Je n'ai pas beaucoup revu ce Abû Magdî, je ne me souviens pas vraiment de sa physionomie. Ce fut notre unique discussion, et son visage se confond désormais avec celui de dizaines d'autres instrumentistes avec lesquels j'ai échangé quelques mots et que je suis incapable de nommer pour la plupart d'entre eux. Ce sont des personnes rencontrées dans l'un des cafés de l'avenue, les membres d'un orchestre que j'ai accompagnés sur les lieux de leur engagement, dans ma voiture ou entassés dans un minibus, en compagnie de la danseuse, à qui on aura respectueusement réservé la place à côté du chauffeur. J'ai surtout côtoyé ces musiciens dans les fêtes de mariage de rue, mais aussi les clubs, les cabarets et les soirées privées. Je les ai pris en photos à certaines occasions, j'ai enregistré leur musique et filmé leur performance. Ils sont pour moi la "rumeur » du métier de musicien, ils m'en donnent une vision pleine mais confuse, m'aidant à en discerner les contours, à approcher ce monde social et la culture spécifique de ceux qui le fréquentent. Mais ils ne faisaient pas partie du « premier cercle», celui de mes informateurs «privilégiés » qui se retrouvent régulièrement dans le petit appartement d'Ahmad Wahdân dans le quartier de Darb al-Ahmar. Une dizaine de personnes auxquelles s'ajoutent les passagers, ceux que l'on ne vit qu'une fois et d'autres qui revinrent de temps à autre dans ce lieu qu'Ahmad appelle la "gare » pour en souligner I'ouverture.

Cependant, justice est ici faite au musicien méconnu puisque c'est à lui que je donne la parole en ouvrant ce texte sur les scènes et les coulisses d'un 
terrain d'Égypte. Je n'avais pas noté grand chose de cette conversation avec Abû Magdî, mais cette petite phrase qui réapparaît aujourd'hui après trois années de fréquentation du milieu est d'une troublante banalité. Car ce qu'il affirme est vrai de toute vie, alors en quoi celle du musicien est-elle spécifique? Quel est le sens de cette disparition? Est-ce le déclin biologique, le tarissement du succès ou la fin de la carrière? S'agit-il d'une remarque désabusée sur les incertitudes du destin des musiciens de mariage à une période particulièrement difficile de leur histoire qui risque de voir la disparition de leur profession sous sa forme actuelle? Quelle vision, enfin, traduit-elle du «drame social du travail » ${ }^{1}$ qui conduit les musiciens à sans cesse négocier leur statut afin de maintenir leur dignité vis-à-vis de leur public et des membres de la société citadine en général?

La réponse à cette interrogation complexe suppose de longs détours que je réserve à une prochaine publication plus complète. Elle est, en revanche, pré-texte à relier l'exploration d'un monde social et de ses territoires à l'acte d'observation, en laissant ainsi l'enquêteur anthropologue apparaître dans le champ des descriptions du «tourbillon urbain $»^{2}$ cairote.

Ainsi, que ce soit au café, au domicile d'Ahmad, dans les différents contextes de la performance - sur l'estrade des fêtes de mariages ${ }^{3}$ ou attablé en contrebas, "posté », le plus souvent caméra à la main, ou encore lors d'enregistrement de répétitions et de concerts - la rumeur du métier se muait en une sonorité plus claire, donnant à entendre la culture des musiciens de l'avenue et, à travers elle, l'une des voix de l'urbanité du Caire.

Je commençais alors de décrire une culture professionnelle et urbaine, ancrée dans des lieux et construite dans un rapport spécifique aux mondes sociaux de la ville, projet qui ressortit au domaine de l'anthropologie urbaine. Celle-ci combine différents points de vue, approches et méthodes pour rendre compte de la complexité des interactions entre des personnes et leurs domaines de significations en ville.

Les fêtes de fiançailles et de mariages (farah, pl. afrâh) de rue constituent la plus grande part des engagements des musiciens de I'avenue Muhammad Ali et sont l'un des lieux privilégiés d'expression des identités citadines. Moment particulier dans l'intimité urbaine des quartiers populaires, elles sont à l'articulation de l'approche en termes de métier et de culture professionnelle des musiciens et de celle qui est centrée sur les mises en scène de la société citadine. Il s'agit d'une situation sur laquelle j'ai concentré des méthodes diversifiées de recueil des données. C'est donc à propos de musiciens, de mariages

1. Everett C. Hugues, 1996, p. 85.

2. J'emprunte cette expression à Ulf Hannerz qui I'utilise pour désigner la façon dont les villes s'ouvrent aux flux culturels et les encadrent sous forme de « tourbillons urbains » (urban swirls) (1992, p. 173-216).

3. J'étais parfois invité par les musiciens à monter sur l'estrade pour filmer. 
et de citadins que je vais expliciter ma relation au terrain ${ }^{4}$. Je distingue trois modalités spécifiques de recueil des données, des méthodes donc, qui donneront au texte un rythme ternaire.

Ce qu'il est convenu d'appeler « observation participante » constitue « I'accroche " privilégiée au terrain. L'enquête est dans ce cadre co-produite avec mes informateurs, notamment Ahmad Wahdân, mais aussi d'autres avec lesquels nous étions engagés dans une démarche compréhensive du monde social des musiciens. Les situations de fêtes qui transforment les rues en arène des visibilités mutuelles se prêtent particulièrement bien à l'enregistrement vidéo. Je m'arrêterai donc sur ce mode d'observation qui permet l'enregistrement de données d'un type particulier. Enfin la matière musicale et le paysage sonore de la fête d'une façon générale sont placés au centre de l'enquête et c'est au moyen des enregistrements audio qu'ils sont recueillis. D'autres moments de performance que je n'aborderai pas ici viennent compléter cette archive anthropologique : répétitions, concerts et collecte de supports musicaux divers (CD, cassettes, vidéos). Ce temps musical est celui des justifications des styles de jeu et des répertoires, ainsi que celui des interactions entre les musiciens et entre ces derniers et leur public dans le cours de la performance.

\section{AVEC LES MUSICIENS : CE NE SONT QUE DES MOTS...}

\section{Le parrainage}

C'est lors d'un séjour au Caire dévolu à l'étude de la langue arabe que se mirent en place les personnages principaux et le cadre d'une enquête qui allait débuter presque dix ans plus tard. J'avais alors rencontré quelques musiciens, à commencer par mon guide et ami Ahmad Wahdân, mais aussi Ali le chanteur devenu plombier ou encore Tal'at, "I'ambianceur " ${ }^{5}$ qui anime les fêtes du son de sa voix sonore. Je les suivais dans leur repère, un café de l'avenue Muhammad Ali, ancien centre emblématique de la musique égyptienne qui s'enfonce chaque jour davantage dans un morne déclin (Puig, 2001). Étant moi-même musicien amateur, j'échangeais avec eux «quelques notes » et idées sur les styles musicaux en Égypte. Sans nul doute la familiarité que j'entretiens avec le domaine

4. Ce qui est dit ici, malgré quelques différences, peut être étendu à d'autres types d'engagements procédant d'un mode semblable de fêtes familiales. Ils sont toutefois plus rares. Il s'agit essentiellement des fêtes de circoncision, des anniversaire et des fiançailles.

5. Nabatshî. Ils sont à l'origine les aides des musiciens dont ils portent les instruments. Ils ont acquis une position privilégiée au sein des mariages depuis que le rite a évolué dans les années 70 pour mettre la collecte des dons monétaires des convives aux familles organisatrices du mariage au centre de la cérémonie. 
musical a-t-elle constitué un facteur important dans l'établissement de solides relations et, par la suite, facilité mon intégration dans le milieu.

De retour au Caire pour un long séjour, retrouvant Ahmad et sa famille, je débute une enquête, avec en tête les quelques lignes écrites par U. Hannerz sur la ville et le travail de terrain dans lesquelles, après avoir affirmé l'utilité de la méthode de l'observation participante, il avance que «le travail de terrain doit être perçu comme protéiforme, s'adaptant sans cesse aux nouveaux contextes en modifiant les procédures établies, s'inspirant de la situation de terrain pour fabriquer de nouveaux outils d'analyse» (1983, p. 381). Ce que I'on peut résumer par la formule « répondre aux injonctions du terrain », ainsi que me le conseillait mon directeur de thèse, Christian Bromberger, lors d'un premier terrain dans le sud de la Tunisie.

Je me donnais alors comme programme de recherche d'observer les pratiques, de suivre les interactions entre les musiciens, avec leur public mais aussi leur voisinage, de recueillir les discours des personnes à propos de leur vie professionnelle... Cela induisait des moments d'observation flottante dans les cafés de l'avenue Muhammad Ali, des situations d'interlocution régulières, I'accompagnement des musiciens dans les différents contextes de leur vie quotidienne et également quelques entretiens longs et personnalisés, en tête à tête, avec ceux des musiciens que je connaissais le mieux, ce qui devait en garantir la pertinence et la profondeur. J'ai effectué la plupart de ces entretiens durant l'automne 2002 après trois ans de fréquentation du milieu, ce qui m'a

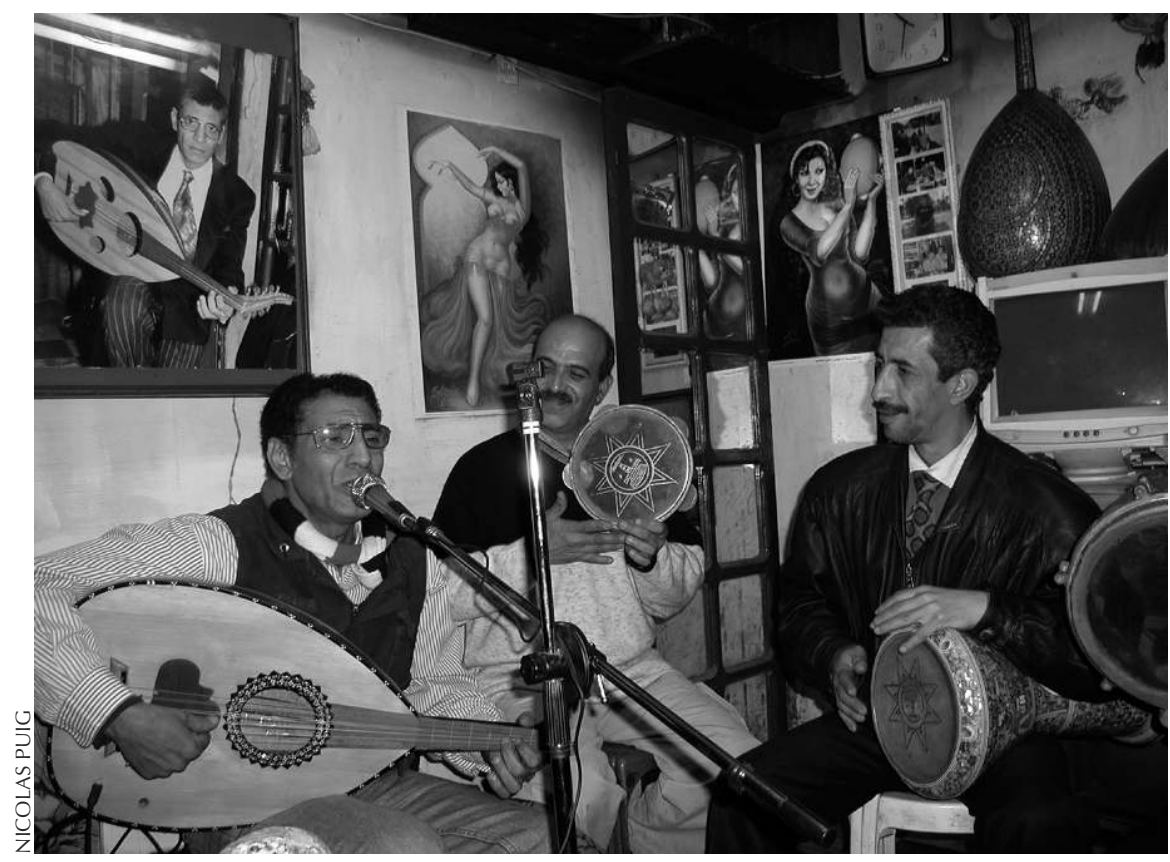

Répétition chez Ahmad Wahdân, 2005 
permis de leur donner un tour personnalisé, adapté à chaque personne et à sa trajectoire dans le monde de la musique.

La méthode qui s'appuie principalement sur la fréquentation assidue des musiciens nécessite en tout premier lieu une « entrée » permettant une intégration au milieu.

Le "parrainage » efficace d'Ahmad Wahdân, chanteur et poly-instrumentiste talentueux (luth arabe, accordéon et piano électrique), figure du milieu des musiciens de I'avenue Muhammad Ali, correspondait au système en vigueur dans les cafés de musiciens où il est nécessaire d'être présenté par un membre de la corporation. Sous son couvert, j'ai pu me glisser en tant que chercheur dans cette petite société bousculée par une modernité agressive.

Cette intégration n'est que relative et pragmatique : elle permet de légitimer la présence du chercheur et éventuellement sa curiosité. On se gardera en la matière de toute naïveté, l'anthropologie contemporaine développant nombres d'analyses sophistiquées, portant sur les interactions entre le chercheur et ceux qui sont objet de la recherche et instituant ainsi les conditions de production de l'enquête en thématique à part entière du champ ${ }^{6}$. Ainsi, je ne tentais pas d'occulter ma présence, soit par la discrétion - en effaçant les traces derrière moi de façon à ne pas perturber le tableau naturaliste dont il s'agissait de rendre compte - soit, à l'inverse, par une intégration totale (devenir l'un d'entre eux pour témoigner « de l'intérieur» de leur condition...). Ce fut plus prosaïque que cela, et c'est dans la construction d'une familiarité ${ }^{\prime}$, la présence répétée finissant par « aller de soi » du fait de la durée de la fréquentation, que je négociais ma position dans le milieu, au coup par coup ou de façon plus pérenne, avec ceux du " premier cercle ». La négociation des rôles, surtout du mien au demeurant, était dans tous les cas un élément récurrent intervenant dans la dynamique des conversations.

Par ailleurs, j'étais bien conscient de travailler avec des personnes exerçant un métier plus ou moins tabou - du point de vue des normes morales à référents religieux - faisant l'objet d'une stigmatisation récurrente de la part des membres de la société conventionnelle et souffrant de ce fait d'un manque de reconnaissance sociale et professionnelle. Cela oriente la présentation de soi, et différentes stratégies étaient alors suivies pour négocier un statut favorable au regard du chercheur occidental auquel, toutefois, il est toujours possible de parler comme à un musicien et, pour certains, à un ami. En règle générale, le monde de la musique étant hiérarchisé, il est compréhensible que l'information et les significations soient peu ou prou filtrées par l'état des relations sociales. De même, le contexte de la conversation influe sur son contenu par diverses

6. Voir Bertrand Masquelier et Jean-Louis Siran (2000) et plus généralement les travaux ressortissant à l'anthropologie de la communication.

7. Vis-à-vis d'Ahmad, il était au demeurant plutôt question de superposer une relation anthropologique à une relation amicale préexistante. 
interférences liées à l'hospitalité, au droit d'aînesse (le plus ancien a la priorité dans la conversation) ou encore au respect des hiérarchies en place, comme dans le cas des entretiens menés avec le président du syndicat des musiciens. Il existe également bien des moments où I'on (se) raconte dans la franchise, où les discussions entre les personnes dévident leur propre logique, sur laquelle la présence oubliée d'un chercheur un peu coi a bien peu d'influence. Des moments de déprise et de neutralisation des enjeux de la "face » - dans ce cas, vis-à-vis des collègues plus que par rapport au chercheur dont l'extériorité au monde social fait que I'on se confie, parfois, plus facilement à lui - au cours desquels diverses considérations peuvent être échangées de façon sincère. De plus, et cela est fondamental dans la démarche présentée ici, l'enquête avec les musiciens ne se réduit pas au recueil de discours sous diverses formes (discussion, entretiens formels, observation flottante...). Les pratiques permettent également d'observer ce que les personnes font et comment elles le font, sans s'en tenir à ce qu'elles disent et ce qu'elles disent de ce qu'elles font... La connaissance des contextes de performance, le suivi des musiciens dans leur engagement permettaient d'ajuster, la plupart du temps, les discours de façon à équilibrer la relation anthropologique.

Dans ma relation avec Ahmad, qu'en d'autres temps on aurait désigné comme un informateur privilégié, j'étais moins gêné par les écueils que la tradition anthropologique ne manque pas d'identifier dans le fait de dépendre en grande partie d'une personne pour mener une enquête ${ }^{8}$, que par son goût et son talent pour manier la parole. La mise en œuvre de ce type de réflexivité correspond à un moment d'ethnologie de la parole, laquelle tente d'intégrer les contextes d'interaction et de présentation de soi sur la production du sens sans prendre la parole proférée comme le témoignage universel d'un univers culturel et social ${ }^{9}$. Mais cet indispensable travail en amont, lié à des enjeux

8. L'anthropologie a bien noté les écueils dans ce recours à un informateur privilégié. Le « danger » existe par exemple d'une sorte d'invention de la culture réalisée à l'intention de l'observateur par l'informateur (cf. par exemple une relation classique d'un travail de terrain par Paul Rabinow (1988) ou, plus récemment, Anne Doquet (1999) qui insiste notamment sur la façon dont les Dogons s'approprient les mythes ethnographiques forgés sur leur culture depuis les entretiens entre Ogotemmeli et Marcel Griaule, pour correspondre à l'image que se font d'eux les visiteurs occidentaux).

9. Il me semble ici que les réajustements se font dans le cours des conversations par le biais de la compréhension que le chercheur a de son terrain; une fréquentation assidue du terrain permet d'évaluer la façon dont le contexte de l'interaction modifie en profondeur l'information échangée. Il est normal aussi que lorsqu'on demande aux personnes de parler d'elles-mêmes, choses qu'en général tout le monde apprécie d'ailleurs, celles-ci se présentent sous un jour particulier, fonction de nombreux paramètres. 
déontologiques de comportement du chercheur face à ses exigences de connaissance, néglige (et c'est d'une façon générale le cas des études réflexives sur le terrain, aussi sophistiquées soient-elles) la composante que j'ai envie d'appeler « ludique » de l'exercice.

Il existe ainsi des terrains plus " prolixes » que d'autres et, bien que l'anthropologue aime à susciter et à délier la parole, peut-être, en effet, une certaine circonspection est-elle de mise avec des spécialistes du "kalâm », hommes de scène, chanteurs et improvisateurs, à l'instar d'Ahmad, animés d'une verve peu commune. II s'agit de faire la part des choses et de laisser aux conversations leurs dynamiques propres, lesquelles n'ont pas forcément à voir avec le compte-rendu objectif de ce qu'est la vie d'un musicien et de la façon dont il évolue dans les différents contextes sociaux. La parole se libère vite et facilement, elle est performance à l'adresse d'auditeurs, elle glisse d'un sujet à l'autre, dans son propre enthousiasme et sa propre dynamique. Elle voyage d'une personne l'autre sans trop craindre les contradictions, les non-sens, voire certains irréalismes parfois. Aspects ludiques de la conversation et rhétorique déployés sont peu reliés à l'établissement d'une vérité, mais jouent un rôle social de négociation des statuts, de partages de codes comme de déliement des imaginations. Il existe dans le milieu, a fortiori chez les chanteurs habitués à la scène et à l'improvisation des longues nuits de fête, une esthétique de la parole et une économie de la conversation. Je propose $d^{\prime}$ en apporter une petite illustration à travers une discussion autour des notions de succès et de prestige, centrales dans le métier, avec celles de honte et $d^{\prime}$ amour $^{10}$.

Vignette ethnographique : le cadre et la photo ou si deux ânes braient ensemble sur un même ton, ils font de la musique

Dans le dialogue ci-dessous, Ahmad intervient pour mettre en relief et expliciter la relation par Mahmûd d'une expérience professionnelle dans un hôtel situé dans une ville de la péninsule arabe. Pour ne pas être trop prisonnier de cette parole, le recueil des discours s'accompagne d'une observation des situations de performance et s'inscrit dans une démarche sur le long terme qui reconsidère en permanence l'analyse des phénomènes étudiés. Seule une bonne connaissance des contextes, c'est-à-dire le fait d'avoir accompagné les orchestres de façon régulière, permet de nuancer le discours sans toutefois le mettre radicalement en cause. La comparaison du cadre et de la photo développée par Ahmad est séduisante, mais elle ne couvre pas l'ensemble des situations de performance musicale. Elle s'applique assez bien aux fêtes de mariage et aux musiques de circonstances en général, mais beaucoup moins à d'autres contextes au cours desquels les instrumentistes sont davantage sur

10. Puig, 2006, b. 
le « devant de la scène» (comme les nuits d'artistes par exemple ou les soirées privées musicales ${ }^{11}$ ). De plus, il existe dans l'ensemble des musiques «populaires» des moments importants d'exécution au cours desquels les instrumentistes " prennent leurs droits » (ya'khdû haqqhum) selon l'expression consacrée. Pourtant, quelque chose est dit sur les relations entre les musiciens, les questions de jalousie et la façon dont se négocie le « capital prestige » de chacun. Le commentaire n'est donc pas faux, au contraire, il est pertinent dans le contexte du récit de Mahmûd. On n'est pas étonné dans I'anthropologie contemporaine par cette affaire de pertinence et de contexte...

\section{Dialogue Ahmad-Mahmûd-Nicolas, Darb al-Ahmar, novembre 2002}

\section{$[\ldots]$}

$M$ - Je faisais un "show » avec la danseuse, je me lève et fais quelques mouvements avec elle, je danse et mets la tabla par terre et je danse en jouant. Je mets la tabla entre mes jambes et je continue à danser et ça plaisait beaucoup aux gens ${ }^{12}$. Mais un des types de l'orchestre lui a dit « il tire la couverture à lui ».

$\mathrm{N}$ - De l'orchestre?

M - Oui de l'orchestre et il lui a dit « il te prend le succès » "biyakhud minik al-succes », tu dois faire attention, tu es la danseuse, c'est toi le numéro (nûmrû). Celui-là il ne doit pas te prendre le succès. Alors elle m'a demandé de ne plus le faire. Mais quand j'ai arrêté, les gens, les clients n'étaient pas contents et le propriétaire du lieu était en colère. Ils pensaient que j'avais décidé de ne plus le faire de mon propre chef. Le propriétaire et les clients ont demandé à la danseuse que je fasse ce numéro mais elle n'a pas voulu et elle a échoué parce qu'elle ne m'a pas laissé faire ça. Elle était supposée rester deux mois, elle est restée vingt-cinq jours et n'a pas terminé son contrat.

$\mathrm{N}$ - Une danseuse nouvelle est venue?

11. Fête organisée par un musicien de l'avenue où se succèdent les «numéros » de collègues destinée à récolter de l'argent sous forme de dons monétaires et mettant en jeu des relations d'obligations réciproques entre les membres de la profession et soirée donnée par une personne au cours de laquelle interviennent un ou plusieurs musiciens.

12. Le numéro proposant une chorégraphie entre l'almée et le percussionniste à la tabla (darbûka) qui la fait danser est courant et fait partie du show de toute bonne artiste. Il implique de la part de l'instrumentiste d'ajouter une dose d'adresse à ses aptitudes musicales. Dans cet hôtel, l'orchestre constitué de personnes qui ne se connaissent peu ou pas du tout n'est pas soudé et les musiciens en situation de concurrence et au statut incertain (il s'agit d'une bonne place que l'on peut perdre facilement) se jalousent. 
M - Oui et je faisais mon numéro avec elle, et elle n'a pas pensé au succès du tout. Mais on avait des camarades pas sympas, ils faisaient des intrigues et des trucs pas bien.

$\mathrm{N}$ - Dans l'orchestre?

$\mathrm{M}$ - Oui,

$\mathrm{N}$ - L'orgue, le oud?

$M$ - Ça pouvait être $n^{\prime}$ importe lequel $d^{\prime}$ entre eux. Le problème est psychologique. "Comment tu te distingues de nous? », mais quand je fais un truc comme ça, je ne réussis pas seul. Les gens disent que I'orchestre est bien et que ce tabbâl est bien. Mais ils vont dire "I'orchestre ", c'est-à-dire qu'ils ne vont pas dire "ce tabbâl» et ils se taisent, ils oublient l'orchestre. Non. Ils disent « cet orchestre » est bien et «le tabbâl » qui y joue est bien. Les autres musiciens ne voulaient pas comprendre ça. Celui qui intrigue et fait ça ne pouvait pas comprendre.

$\mathrm{N}$ - Et le reste des gens de l'orchestre était égyptien?

$M$ - La plupart

$\mathrm{N}$ - Comme toi, ils sont venus d'Égypte pour travailler?

$\mathrm{M}$ - Oui, ils sont venus pour travailler, on est un groupe qui travaille ensemble

$\mathrm{N}$ - Pourquoi t'ont-ils fait des problèmes?

$M$ - À cause du succès, qui va monopoliser le succès et qui va avoir les faveurs du public et les applaudissements. Comment ces applaudissements sont pour moi, et on m'apporte du champagne, et pas à elle...

$\mathrm{N}$ - Qui t'apporte ce champagne?

$M$ - Les clients

$\mathrm{N}$ - Ils te payent une bouteille de champagne parce que tu joues bien de la tabla...

M - Oui, le client paye le champagne pour les tabbâls. Ils disent « maître please, donnez du champagne pour Mahmûd » et un autre client offre du whisky. Il y avait des gens que ça énervait.

$\mathrm{N}$ - Ils étaient jaloux ou quoi?

M-Oui

$\mathrm{N}$ - Mais, comment cela arrive-t-il?

Ahmad qui était resté silencieux jusque là intervient :

A - Je suis au travail et j'ai du succès, les gens viennent me voir et me donne beaucoup d'argent, ils sont très contents de moi. Un chanteur vient et fait un numéro, il sent qu'il est faible par rapport à I'orchestre. Il commence à dire du mal de celui qui organise le travail et de l'orchestre, il dit que cet orchestre est nul. Celui qui chante et celle qui danse sont ceux qui sont devant le public directement. Les musiciens sont comme un cadre, ils encadrent le numéro, tu ne peux 
pas dire que le cadre est mieux que la photo. En premier, tu regardes la photo qui est le numéro, la danseuse ou le chanteur, ça c'est la photo. Les musiciens sont juste les cadres. Quand le cadre brille et est plus beau que la photo, la photo se met à le jalouser. Elle va interagir avec le public. Une chanteuse peut être faible avec un orchestre excellent, mais elle peut faire douter le public sur l'orchestre et dire c'est parce qu'ils ne me donnent pas la hauteur à laquelle je suis à l'aise pour chanter; c'est eux les responsables, ils ne savent pas m'accompagner : " c'est un âne, c'est quoi ça, il ne sait pas jouer», mais le public la croit, elle, il ne croit pas les musiciens et les clients aussi qui amènent I'orchestre la croit, elle. Alors ils disent « amène la danseuse et n'amène plus cet orchestre ou ce chanteur et amène un orchestre qui accompagne bien le chanteur ou la danseuse ». À cause de ça, quand je veux travailler je dis " quel chanteur je vais accompagner? ». C'est la première chose, avant le travail. Et c'est l'une des causes qui expliquent mon ancienneté sur la place. Je ne travaille pas pour n'importe quel numéro : je refuse de travailler avec $n^{\prime}$ importe quel numéro dans le souk. Je sens qu'il n'y a pas de numéro qui mérite que je reste derrière lui. II n'y en a pas. II faut que le numéro me plaise pour que je le fasse réussir et que je $\mathrm{m}^{\prime} \mathrm{y}$ investisse et que je $\mathrm{m}^{\prime} \mathrm{y}$ fonde complètement. On devient ensemble une même chose. Lui avec moi et moi avec lui. Les deux se complètent. Mais que je le dépasse ou qu'il me dépasse, non, ça ne va pas si je sens qu'il me manque quelque chose, il faut qu'il y ait deux énergies complémentaires. Très simplement, si deux ânes braient ensemble sur un même ton, ils font de la musique. Prends une mauvaise chanteuse et un mauvais orchestre. Les deux plaisent aux clients. Et ils sont très bien. Si un bon chanteur est avec un bon orchestre c'est pareil. II faut qu'il y ait un bon équilibre, une complémentarité. Il y a des très bons musiciens qui, sachant que cette chanteuse est mauvaise, deviennent eux-mêmes faibles comme elle, pour ne pas lui gâcher le travail. Ils descendent leur niveau au sien. Et ce genre de choses, ça ce ne se fait pas comme ça. II faut qu'il y ait de I'affection, de I'harmonie. Pour que je reste avec les musiciens, il faut que je m'entende bien avec eux avant même d'accorder mon instrument. Je m'accorde à eux, je suis sympa avec eux afin qu'ils ne me couvrent pas après avec leur instrument. Lui, il joue un truc et moi un truc et tous les deux on fait quelque chose de bien. Je le mets en valeur et lui le fait avec moi. Mais on ne se dispute pas, musicalement. Le son de l'orgue est fort et près de moi je trouve un violon avec un petit son, je vais le couvrir, le tuer musicalement? Je le laisse comme ça, sur la photo ou je le jalouse et ne laisse pas jouer avec moi. Si je fais ça, moi, je suis un musicien mauvais de l'intérieur, un musicien pas bien. Il faut que je sois en harmonie avec les autres musiciens de la même façon que j'accorde l'instrument avec lequel je travaille. 
Cette longue explication d'Ahmad, parmi de nombreux discours de son cru ou émanant d'autres personnes, montre bien, me semble-t-il, la séduction et les dangers d'une parole déliée. II ne s'agit pas de la brider, au contraire (si ce n'est dans les cadres très spécifiques d'un entretien formel), mais de la considérer pour ce qu'elle est. L'interprétation d'Ahmad procède à la fois de codes socialement partagés quant à la place du chant et de la danse par rapport à une musique purement instrumentale conçue comme secondaire (dans le contexte des musiques de fêtes), tout en apportant une lecture personnelle un peu idéalisée de la pratique professionnelle placée sous l'égide de l'harmonie et de l'affection. En effet, de l'avis général et y compris de celui $\mathrm{d}^{\prime}$ Ahmad qui ne se produit quasiment plus dans les fêtes de rue, les mariages de rue n'autorisent pas vraiment ce genre de recherche, même s'il arrive que I'ambition artistique se fraie un chemin dans l'ambiance sonore de la cérémonie. Il reste à tester cette formule du cadre et de la photo et ses implications par une observation des orchestres en situation de performance, puis à en rediscuter avec Ahmad et Mahmûd. L'enquête se présente ainsi sous la forme $d^{\prime}$ une co-production qui suit les multiples chemins de significations contextualisées que suivent les musiciens dans le cadre de leur vie professionnelle.

\section{Observation flottante : un extrait détaché}

Aux côtés d'entretiens formalisés par l'enregistrement, l'enquête se construit également aux abords de dialogues décousus. Du point de vue de la pratique de recherche, le plus souvent, " passer le temps », ou plutôt " passer du temps », ne revient pas à le perdre. Le décalage ethnographique par rapport à des situations directes d'enquête est essentiel à ma pratique et à ma façon de concevoir la relation anthropologique. Cette présence assurée de façon régulière n'est autre qu'une forme de participation sociale qui prend, certes, des formes un peu spécifiques sur les terrains urbains. En effet, la diversité des mondes sociaux en ville permet au chercheur une forme de présence discontinue; résidant dans une autre partie de la ville, menant, éventuellement, une vie sociale, familiale, en tout cas affective, amoureuse, peut-être, occupant parfois un bureau au sein d'un institut, il remplace l'intensité $d^{\prime}$ un terrain à plein temps par une fréquentation régulière mais intermittente, prenant place sur de larges plages temporelles.

$C^{\prime}$ est au cours de longs moments d'observation flottante que s'élaborent certaines des pistes de recherche parmi les plus intéressantes et que sont collectées nombre d'informations importantes, dénotant par rapport aux réponses stéréotypées des situations d'enquête plus formelles ${ }^{13}$. À titre d'illustration,

13. Dans le contexte un peu différent d'une société "surpâturée » par les ethnologues, Anne Doquet indique que c'est en jouant aux cartes qu'elle a pu dépasser les blocages de ses entretiens formels qui n'appelaient que des réponses toutes faites de la part de ses interlocuteurs (1999, p. 315-316). 
je livre ici un extrait issu d'un carnet de terrain rendant compte d'un moment d'observation flottante avenue Muhammad Ali en 2004.

\section{Au café Sa'd al-Sawa lors de l'aïd 2004 avec Ahmad}

Au cours de ce mois, peu de travail pour la plupart des musiciens, durant le mois de Ramadan, il y a peu de mariages. L'avenue s'éveille de la léthargie qui fut la sienne ces dernières semaines. Un joueur de tabla vient s'asseoir quelques instants avec nous. Lui a pu travailler tous les soirs lors des repas de rupture du jeûne dans le restaurant du nouveau jardin de l'Aga Khan à la citadelle (100 L.E. le menu, réservé aux Égyptiens relativement fortunés et aux touristes).

La discussion tourne autour de la jeune génération incapable de remplir de tels emplois : " ceux qui travaillent dans le souk maintenant jouent sur 2 ou 3 gammes simples : majeur, nahawand, rast. Ils ne connaissent pas le reste, ils n'ont pas étudié ». À ce moment, un jeune chanteur nous interrompt pour demander à Ahmad comment chanter " yâ halâwat al-dunyâ ». Il parle de turâth, comme c'est souvent le cas pour une chanson dont on n'est pas trop sûr du compositeur, on parle de turâth, chanté par... en l'occurence Sayyid Mikkawi.

Les morceaux sont autant connus par leur interprète que par leur compositeur [tient au mode d'apprentissage par l'oreille? Exemple des cassettes ou de la radio : Umm Kalthum qui chantait chaque semaine à la radio plusieurs fois le morceau, car elle savait que tous les musiciens d'Égypte l'écoutaient pour reprendre le morceau m’a raconté Samiha al-Kureishi, une ancienne de l'avenue (aujourd'hui décédée); elle avait ajouté que ce type de musicien était zay al-karbun (comme le papier carbone)].

Au café, un sosie de Sha'bân [Sha'bân 'Abd al-Rahîm, chanteur « populaire » cairote] s'assoit non loin de nous et nous salue avec nonchalance.

Ahmad : «Avant, pendant l'aïd, c'était impossible de voir des musiciens sans emplois, ça durait les trois jours de fête ».

Vers les 4 heures de I'après-midi, I'ambiance monte, le trafic sur I'avenue est plus dense et les cafés se remplissent. Une ambiance de fébrilité s'instaure.

«Le musicien de Muhammad Ali est un artisan, il supporte le travail, peut enchaîner le travail toute la nuit »: Salâma qui s'est assis avec nous.

Un musicien désœuvré passe, il semble nerveux, cravate jaune pour costume sombre, sort visiblement de chez le coiffeur, dernière coupe à la mode, un peu long derrière, court devant et légèrement bouclé (apparence chic populaire propagée par Sha'bân).

"Ay maslaha ana ma'ak» [n'importe quel boulot, je suis avec toi] lui dit Salâma: "Je ne sais pas ce qu'il se passe, mais il y a quelque chose quelque part », il ajoute avec un soupir : " I'avenue c'est fini ». 
Il jouait du oud, mais s'est mis à l'orgue électrique pour le souk. $\mathrm{C}^{\prime}$ est avec cet instrument qu'il gagne sa vie.

Peu de travail, Ahmad est triste pour les musiciens : " Ceux qui sont là sont en urgence ». Celui qui ne trouve pas de travail est désespéré de rentrer sans argent surtout pour la fête. Le café ou nous sommes est " sha'bi shwayya » [un peu populaire] par rapport à halâwathum [café d'en face]. Ahmad malgré son nouveau prestige [grâce à une série de concerts un peu prestigieux] n'a pas changé. Selon lui, quand on est dans un café, il faut y rester. Devenir un habitué pour qu'on sache où trouver la personne. Par exemple, s'il prend une avance et disparaît dans la nature. Ici, on sait où le trouver n'importe quand.

Les musiciens se pressent, se saluent, s'interrogent mutuellement sur les engagements du soir. Un vieux trompettiste avec un sac en toile comme étui, des percussions dans des étuis troués et rapiécés. Jimmy essaye de m'expliquer comment émettre des 1/4 de tons à la trompette, en gros " $c^{\prime}$ est à la gueule », comme on dit. Tout le monde s'empresse autour des impresarios qui rassurent et jouent du téléphone portable avec ostentation. Ce soir, il n'y aura pas du travail pour tout le monde renchérit Salâma.

[...]

Cet extrait de carnet ne fut pas écrit sur le moment. II s'agit de « notesmémoires ${ }^{14}$ qui se différencient de celles rédigées directement dans le cours de l'interaction avec les informateurs. Seuls sont consignés de façon discrète les sujets abordés, les événements, les choses observées, mais la priorité est donnée à l'interaction et à l'observation : le fait d'écrire ne poserait pas en soi un problème, mais donnerait un ton beaucoup plus « officiel » à ma présence. Ce n'est que dans un second temps que les notes sont reportées par écrit avec une première série de commentaires et de mises en relation des informations. En plus de données diverses sur le métier, il importe de tenter de rendre compte des ambiances dans un souci de description phénoménologique. Avec les apports d'autres méthodologies plus "directrices », des bribes d'observation flottante viennent ainsi au fil des carnets témoigner de l'expérience professionnelle comme "existentielle » des musiciens. Parmi ces autres méthodes, il y a le recours au film dans les moments de performance.

14. Jean Copans citant R. Sanjek et S. Ottenberg distingue les notes de terrain des notes de tête ou notes en mémoire (headnotes) (2002, p. 98). 


\section{SCÈNES ET COULISSES DES MARIAGES DE RUE : REGARDS AU CENTRE, REGARDS ÉGARÉS}

\section{Carnets vidéos}

Institutions anciennes dont il est question dans les règlements d'urbanisme du $\mathrm{XIX}^{\mathrm{e}}$ siècle $^{15}$, les mariages de rue représentent la principale source de travail des musiciens de I'avenue Muhammad Ali. Ils sont qualifiés de baladî, terme qui atteste de leur caractère local et populaire et les distingue ainsi de mariages afrangî, étrangers (occidentalisés), mais aussi «chics » qui se déroulent dans les salles des fêtes des clubs et des hôtels. Une portion de rue ou de ruelle est appropriée pour la tenue de la fête, créant une incise privative dans l'espace communautaire de résidence. De grandes tentures délimitent l'espace dévolu à la fête tout en laissant un cheminement minimum pour les passants. Un vidéaste est à l'œuvre lors des cérémonies, enregistrant notamment de longs plans des mariés trônant sur des chaises surélevées capitonnées de rouge. Il n'y a donc rien de particulièrement étonnant à ce qu'une seconde caméra accompagne la première ou à ce que des photos soient prises. Au contraire, cela est souvent proposé si d'aventure je n'ai pas prestement sorti mon matériel. Ce sont des cérémonies destinées à être filmées et enregistrées et la plupart des convives ont fait des efforts d'apparence. Aussi est-ce souvent sous la bienveillante autorité d'un homme de la famille que je suis conduit jusqu'aux mariés pour me présenter, puis les filmer ou les photographier tandis que c'est en parfait étranger que j'avais débarqué à la noce dans le sillage des musiciens.

Le fait d'avoir en main un appareil photo ou une caméra permet donc de se déplacer dans la fête de façon légitime, d'occuper plus librement l'espace et de s'affranchir un peu des règles de la bienséance. Cela autorise à prendre des «postes d'observation » qu'il est malaisé d'atteindre autrement, les invités se déplaçant peu, chacun restant à la place qui lui a été attribuée. Cela

15. Le Tanzim dispose ainsi dans son arrêté du 22 juin 1896 : «À I'occasion des mariages, les particuliers pourront être autorisés à occuper la moitié de la largeur de la voie publique, lorsqu'ils en feront la demande en payant les taxes prévues à l'art. 13 dudit règlement. - L'administration se réserve dans tous les cas, de ménager dans les voies occupées, un espace libre suffisant pour assurer la circulation publique. Elle pourra même refuser les autorisations qui lui seraient demandées pour occuper les rues très fréquentées, telles que : le Mouski, Sharia-el-Nahassin, Sharia-elSoukkarieh, Sharia-el-Ghourieh et autres. Les bénéficiaires de la tolérance fixée au dernier alinéa de l'art. 1er, ainsi que ceux qui obtiendront des roksas [autorisations] suivant les prescriptions des paragraphes a et b ci-dessous ont l'obligation de réparer les trottoirs et les chaussées s'ils ont été dégradés ou endommagés par les installations provisoires et ce immédiatement après l'enlèvement de ces installations sous peine d'y être contraints selon la loi. » (Lamba, 1911). 
permet aussi d'occulter un peu sa présence personnelle, en offrant une sorte de refuge dans des contextes où, une fois " démasqué » en tant qu'occidental, on devient l'objet de multiples sollicitations plus ou moins insistantes; être équipé d'une caméra permet de se libérer des contraintes de sociabilité sans contrevenir aux convenances.

Toutefois, les acteurs de séquences de la fête présentant des aspects transgressifs se prêtent, logiquement, moins volontiers à l'enregistrement. C'est le cas des préparateurs de narguilés de petite taille appellés gûza dans lesquelles ils mélangent le tabac à la mélasse et la marijuana apportées par les invités. De même, les tablées d'hommes buvant de l'alcool préfèreront que ma caméra, à I'instar de celle du vidéaste professionnel qui consacre un plan court pour chaque invité, laisse hors-champ les bouteilles de bière, plus rarement de whisky. Il est malvenu de filmer le coin des femmes, à l'exception des moments de la cérémonie au cours desquels l'accent est mis sur les mariés qui sont alors entourés par le public féminin. Entre les moments intégrant un minimum de « mise en scène ", qui doivent être filmés dans la logique de la construction du souvenir des familles et de la préservation de la dignité des invités et les situations que l'on préfère voir laissées dans l'ombre, le chercheur vidéaste a rapidement fait le tri. Et si sa sagacité est mise en défaut, et qu'il est pris à filmer ce qui ne doit pas l'être, il lui sera suggéré, poliment mais fermement, de tourner l'objectif de sa caméra ailleurs. Il lui faudra donc bien faire la différence entre les coulisses et les scènes de la fête, et la liberté surveillée dont il bénéficie grâce à sa petite caméra numérique lui permettra, à titre d'exercice ou de travaux pratiques, d'éprouver les limites au regard qu'il n'aurait pas déjà, tout seul, identifiées.

Outre cet aspect «stratégique » d'occupation de l'espace et d'expérimentation de la compréhension de la situation par le chercheur, le film et la photographie sont, bien entendu, un moyen efficace d'enregistrement des discours publics et d'observation des positions des personnes, des regards et des interactions durant la fête qui viennent seconder le regard direct. Ils permettent de constituer une archive anthropologique et de fournir des données qui, une fois fixées sur des supports relativement durables (cassettes DV par exemple), autorisent plusieurs visions de la fête et de son déroulement.

Le film prolonge le carnet de l'anthropologue en choisissant différentes séquences de la fête : les félicitations du nabatshî adressées aux invités débarquant à la noce ou à de généreux donateurs dont le nom est scandé au micro, l'arrivée discrète, sur le tard, d'un personnage entre deux âges que l'on retrouvera quelques instants après sur la scène, en train de danser aux côtés de l'almée, privilège dénotant un statut élevé, ou encore un esclandre entre deux personnes qui, au grand désarroi de l'ambianceur, s'arrachent le micro pour répandre leurs invectives dans l'espace sonore de la fête et prendre à témoin le public de la noce comme l'ensemble du quartier, etc. Le film dans le contexte d'une enquête anthropologique de terrain constitue en lui-même un exercice spécifique d'observation et de recueil des données, un " carnet vidéo » qui fixe sur la bande magnétique les différentes séquences de la cérémonie saisies au gré du regard du chercheur. 


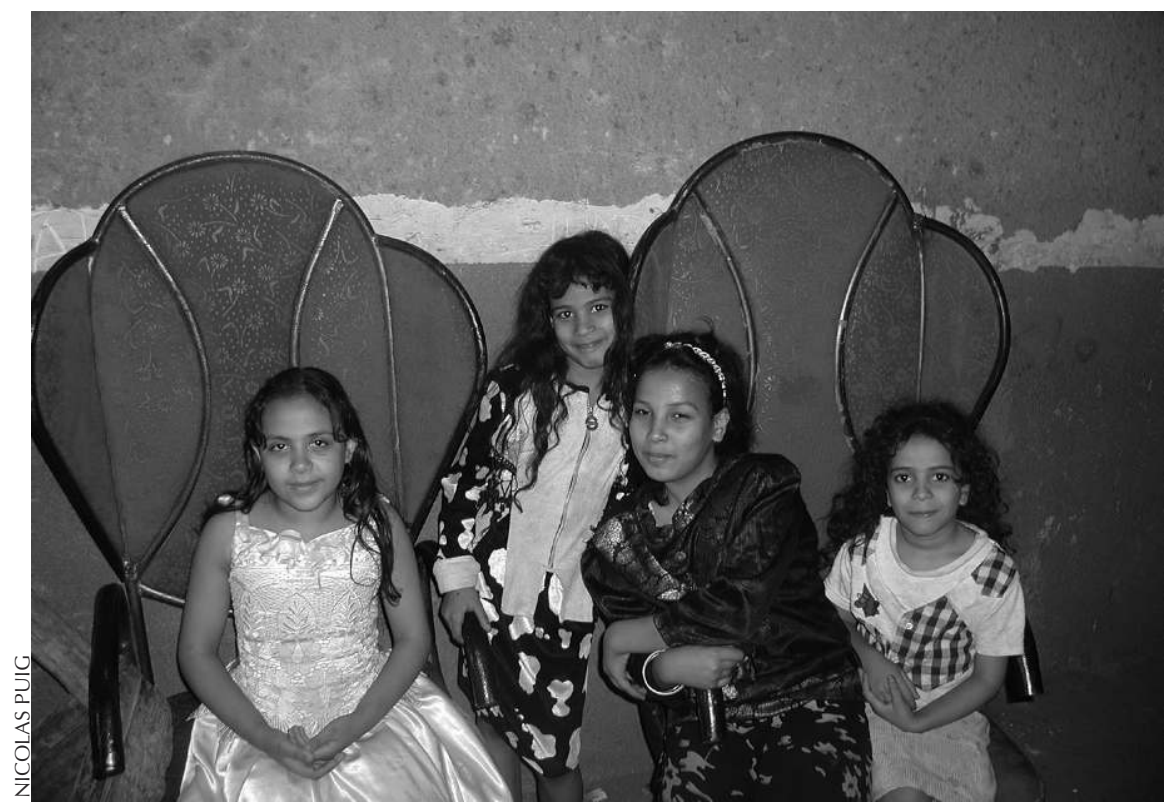

Fin de soirée, les enfants posent sur les «trônes » des mariés qui ont quitté la noce, 2003

\section{Ordres et désordres de la fête}

L'arrivée dans le farah constitue un moment d'intensité cérémonielle, au cours $d^{\prime}$ 'un cheminement en direction de l'estrade sur lequel se concentre une bonne part des éclairages de la fête. Elle se fait avec faste par une allée ménagée entre les tables et les chaises. Le nabatshî, interrompant la musique, salue longuement de son nom le nouvel arrivant à la noce, annonçant le plus souvent son titre (ou lui en attribuant un) et son quartier. C'est un accueil important, dénotatif de la respectabilité de l'invité qui, prestement pris en charge, est accompagné puis installé en fonction de son statut et des relations qu'il entretient avec les familles des mariés, les places de choix étant à proximité immédiate de l'estrade.

Dans l'espace du farah, les femmes, séparées des hommes, sont disposées près du trône des époux, à l'écart, loin de l'estrade, en général dans l'angle mort des regards masculins tournés vers les musiciens et la danseuse. II s'agit d'une sorte de coulisse depuis laquelle se prépare parfois l'arrivée sur scène des mariés pour un moment de danse lors de la soirée. Elle devient une scène le temps de la remise, en début de soirée, des bijoux que le marié offre à sa promise (chabka).

Ailleurs dans l'espace, la disposition dramatique des lieux est soulignée par la présence d'une estrade, masrah. II n'y a pas de coulisses en propre associées à cette estrade, mais plutôt une arrière-scène, voire des endroits de cette scène où I'on prend davantage ses aises. II suffit de peu de choses pour 
cela et il n'est pas nécessaire de sortir du cadre des visibilités mutuelles. C'est là, me semble-t-il, un effet d'hospitalité résultant de la densité des quartiers populaires. L'hospitalité dans le Caire populaire tient, en effet, à la présence de bienséances qui gouvernent les situations d'attention à l'autre et d' « inattention civile » pour que chacun vive sa vie dans la proximité d'autrui ${ }^{16}$. Ce qui n'empêche évidemment pas l'existence d'un fort contrôle social, notamment appliqué aux comportements moraux.

Tandis que la maîtrise des impressions est un élément central de la fête de mariage, la photo présentée ci-dessous laisse apparaître l'aspect négligé du niveau inférieur de l'estrade, lequel suggère que ce n'est pas là que doit se porter le regard. On glissera donc sur les bouteilles de bière reposant couchées dans le voisinage des mégots de cigarette, les pieds déchaussés, les housses d'instruments jetées en vrac et les enchevêtrements de câbles électriques omniprésents. Bien que située au niveau même des regards des convives, cette partie inférieure de l'estrade montre par son laisser-aller que la division scène/ coulisse est davantage une question d'interaction entre les personnes que de propriétés intrinsèques des espaces. De même, sur l'estrade, les danseuses, dès qu'elles se tiennent en retrait, sont en général couvertes conformément aux normes partagées de la pudeur. Elles jouissent ainsi d'un moment de détente et, s'il ne sera jamais totalement dérobé au regard du public, notamment des plus jeunes, ce retrait apparaît déjà comme une coulisse. Ce regard « sans vergogne » des plus jeunes (mais aussi d'autres) constitue, par ailleurs, une manifestation du droit que s'accorde l'autochtone "à ne rien cacher de sa curiosité », selon les mots d'Isaac Joseph définissant " l'épreuve majeure de l'étrangéité » (1997, p. 137). Ce regard nous rappelle que la fête se déroule au milieu d'une communauté de résidence, chez les habitants de la rue nécessairement placés aux premières loges.

Le farah constitue un moment privilégié de la présentation de soi, de l'évaluation et de la reconnaissance de sa position dans la société locale dans un lieu qui ménage des possibilités transgressives. Les comportements, les gestes, les déplacements des corps, les regards, etc. captés par la caméra en témoignent. L'ordre social du mariage se caractérise ainsi par une mise en scène de la société citadine et de ses identités. Ce qui est dit dans la fête ressortit pour partie à l'ordre des territoires urbains à travers l'évocation des noms de quartiers et de leurs qualités supposées. L'annonce du nom des habitants résonne dans l'espace sonore, conférant une reconnaissance publique de la respectabilité et du prestige de ceux dont le patronyme est scandé.

Cette prise en charge est autant un acte d'hospitalité qui complète celui, sonore, de l'orchestre, que le reflet d'un contrôle accru sur un espace ouvert de manière à maintenir les conditions de la pratique ludique et festive. La tension liée à l'intrusion d'étrangers se traduit notamment par une certaine

16. Joseph, 1997, p. 137. 
fébrilité des hommes de la famille invitante. Des débordements dus à un trop plein d'excitation (et d'alcool) peuvent se produire et entraîner des situations délicates lorsque, par exemple, des coups de feu sont tirés en l'air, des danses trop lascives et inconvenantes avec la danseuse exécutées sur l'estrade ou encore le micro monopolisé par un invité squattant la scène. Les incidents ne sont pas rares et les ambiances peuvent parfois basculer vers un désenchantement profond accompagné d'un douloureux rappel du principe de réalité. Aussi les ordres que manifestent les fêtes de rue, ordre collectif et ordre interactionnel, sont-ils bien éloignés du désordre que l'on prête généralement à ce genre de festivités et, à l'instar du carnaval, « la fête n'est qu'exceptionnellement un prélude à la révolte [...] ${ }^{17}$.

Ces contextes de performance se caractérisent par la recherche d'une saturation sensorielle dans laquelle la musique n'est qu'un des éléments constitutifs du paysage sonore. L'analyse de ce paysage très particulier des mariages de rue et de la production musicale constitue le troisième mouvement de restitution de l'enquête qui s'appuie davantage sur l'ouïe que sur la vue.

\section{LE PAYSAGE SONORE DES MARIAGES DE RUE : ÉCOUTER POUR ENTENDRE}

\section{Les marqueurs de la cérémonie}

Si le regard est considéré comme le sens privilégié de la vie urbaine ${ }^{18}$, il n'échappera à nul visiteur du Caire que le son a quelque chose à y voir également. L'écologie sonore de la ville surprend, en effet, ceux qui n'ont pas intégré physiquement sa norme auditive grâce à une longue fréquentation de ses espaces privés comme publics. Il est donc assez logique que le son des fêtes de rue, porté à des niveaux de décibels douloureux, ait pour effet de rendre toutes les tentatives de discussions fort malaisées.

Dans ce cadre, I'enregistrement audio constitue une méthode appropriée pour isoler puis travailler la matière musicale et sonore, même s'il s'avère délicat, compte tenu de la qualité des sons émis. De fait, je n'ai effectué ce type d'opération que dans des contextes spécifiques de production musicale de façon à isoler un matériau de bonne facture. J'ai procédé ainsi à l'enregistrement de répétitions, de concerts publics, de soirées privées données au domicile d'une personne et, également, d'un CD de quatorze titres en attente de production, à l'occasion d'un passage en studio réalisé en mars 2005. Outre leur intérêt artistique, ces données sont utiles pour travailler sur les répertoires,

17. Constant-Martin D., 2000, p. 178.

18. Simmel écrivait il y a plus d'un siècle que : "Les rapports des hommes dans les grandes villes, si on les compare à ceux des petites villes, sont caractérisés par une prépondérance marquée de l'activité de la vue sur celle de l'ouïe » (1981, p. 230). 


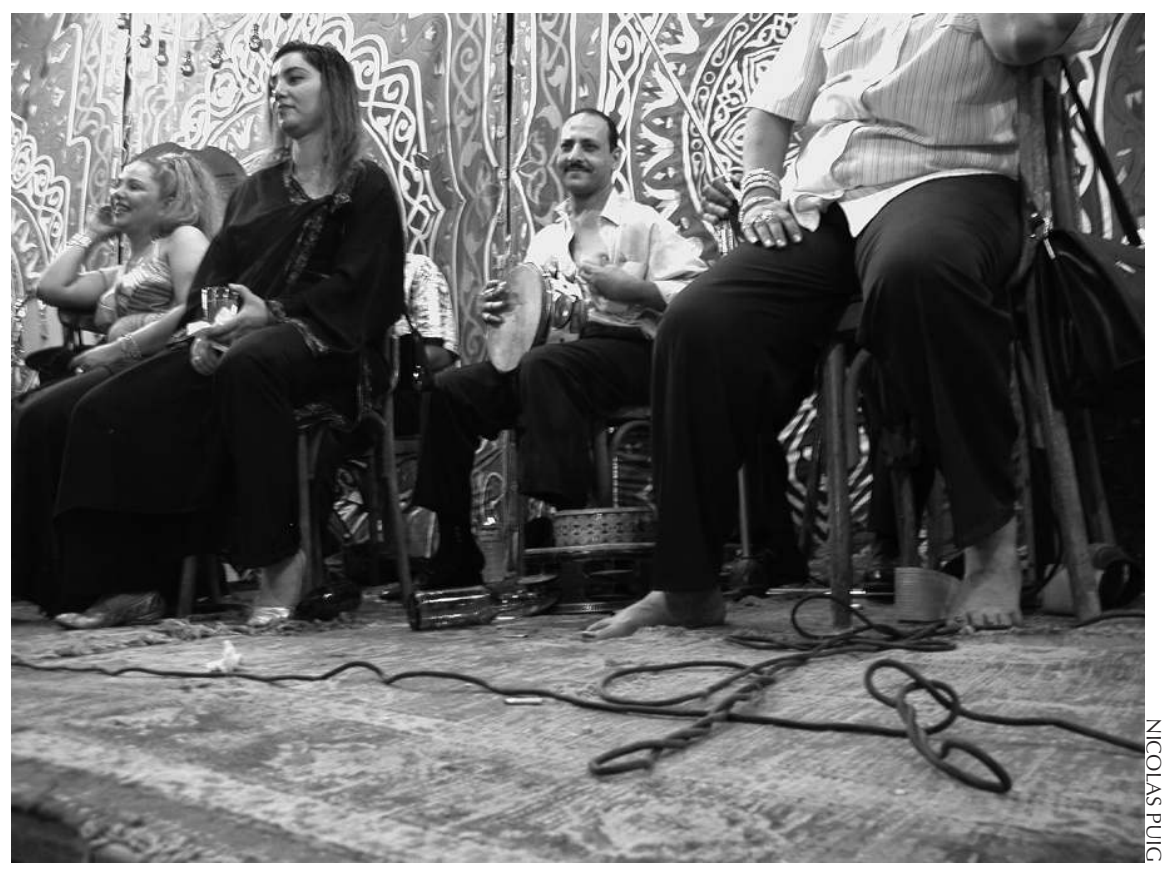

Scènes et coulisses sur l'estrade, 2003

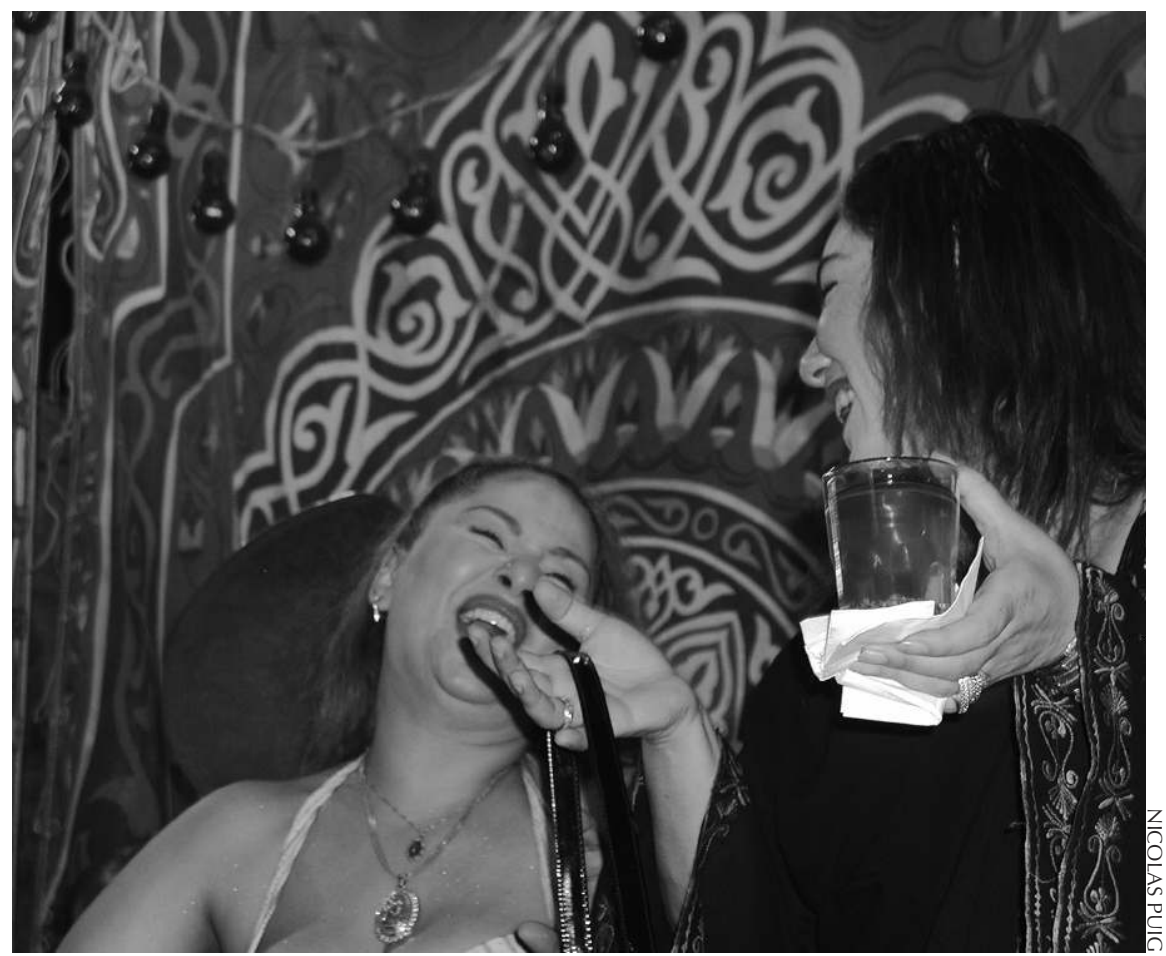

Prise d'intimité dans l'aire des visibilités, danseuses sur l'estrade entre deux prestations, 2003 
les styles, les paroles et finalement les interactions entre musiciens dans le cadre professionnel. En revanche, le paysage sonore du mariage de rue est très spécifique et probablement très difficile à fixer de façon satisfaisante sur un support audio. C'est donc par l'intermédiaire du micro de ma caméra numérique que j'ai travaillé sur les sons de la cérémonie. Ceux-ci se caractérisent par les effets, la puissance et son corollaire, la saturation.

Tout d'abord, l'ambiance sonore d'une fête de mariage identifie sans ambiguïté la cérémonie en la distinguant notamment des fêtes sacrées que sont les

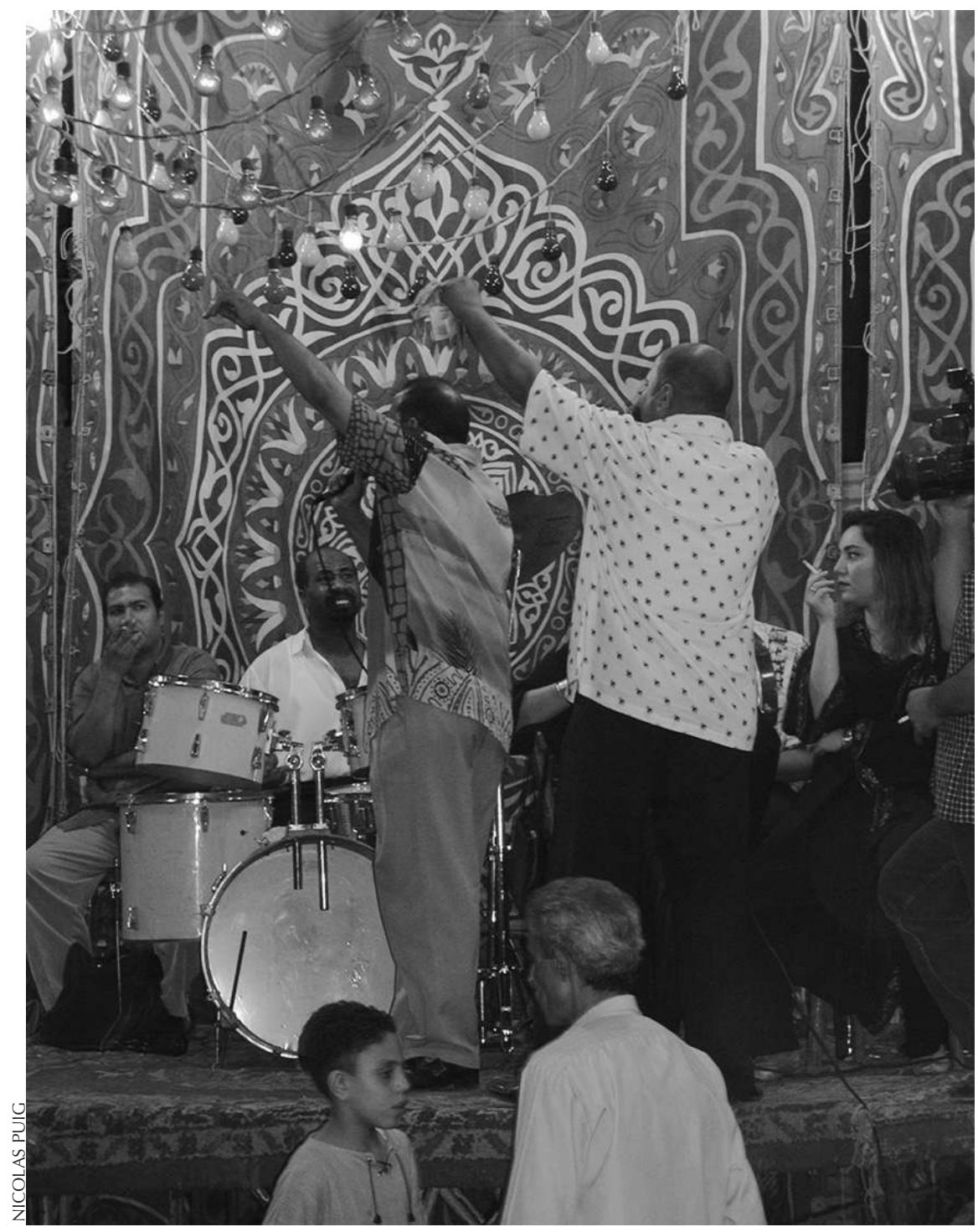

Deux nabatshî accueillent un invité, 2003 
mawlid. Cette différence ne provient pas du matériel d'amplification, qui est similaire, mais des effets utilisés. L'effet « chorus » qui donne au son un écho (la phrase est répétée plusieurs fois en s'amenuisant) est le marqueur sonore du mariage de rue. L'écho permet de meubler l'espace sonore, puisqu'il n’y a en général dans les mariages de rue qu'un seul instrument mélodique (orgue ou accordéon), les percussions et la ou les danseuses. Cela donne ainsi plus de présence à l'orchestre et aux voix des chanteurs et des nabatshî. Dans les mawlid, l'effet privilégié est la « reverb » qui procède du même mécanisme que l'écho, mais dont le temps de répétition n'est pas audible. Une sonorité particulière se dégage ainsi et l'oreille perçoit un son qui semble résonner.

L'espace sonore de la fête se caractérise également par la saturation des sons, musiques et voix des « ambianceurs » qui jouent un rôle majeur dans le paysage sonore par leurs discours rythmés et parfois rimés. Le son est amplifié au maximum des possibilités techniques, ce qui produit une saturation du fait de la faiblesse technologique des matériels employés, la plupart du temps des imitations d'amplificateurs de marque italienne (Montarbo) qui sont distribués dans les magasins de vente et de location de sounds. Ce son sur-amplifié suscite des critiques et des plaintes de la part de ceux qui ne participent pas à la noce et qui ressentent une atteinte perceptive insupportable du fait de leur situation d'extériorité. Le voisinage fait, au demeurant, l'objet d'une attention particulière des nabatshî qui ne manquent pas de le saluer régulièrement de façon à s'en concilier les bonnes grâces et éviter ainsi d'intempestifs appels à la police.

L'appareillage sonore masque bien souvent la faiblesse des orchestres et des voix des chanteurs en leur accordant une présence sonore qui contraste avec la qualité musicale de leur jeu. Porté par cet ample courant sonore, le chanteur est plus à l'aise, de même que l'organiste qui dans les mariages peu fortunés est souvent un jeune en cours d'apprentissage se satisfaisant d'une modeste rétribution. Toutefois, les musiciens ne manquent pas de le souligner, cette ambiance sonore est désirée par les organisateurs du mariage et reconnue par le public comme une propriété festive de la cérémonie à laquelle il est sensible. Une esthétique de la saturation sonore s'est ainsi développée tandis que se diffuse massivement l'orgue électrique, instrument amplifié qui permet toutes sortes d'effets et de sonorités, et dont l'apprentissage serait moins ardu que celui de l'accordéon, par exemple, qui le précéda comme instrument mélodique des fêtes de rue.

La musique est assujettie aux annonces des nabatshî. L'élément fondamental de l'animation et l'enjeu du mariage réside, en effet, dans la nu'ta. Il s'agit de dons monétaires faits dans le cadre d'obligations réciproques à I'adresse des familles organisant le mariage ainsi qu'à l'orchestre ${ }^{19}$. Ces dons

19. Plusieurs possibilités de distribution de cet argent entre organisateur du mariage et musiciens existent. De nombreux conflits intervenant à la fin de la fête sont liés au partage de la nu'ta. 
sont généralement consignés par écrit et, le moment venu, il appartiendra aux membres de la famille organisatrice de faire de même lors du farah de I'une des personnes présentes ou de l'un de ses proches. Ceci est un élément d'explication de l'emphase avec laquelle le nabatshî accueille les invités à la noce, laquelle est soulignée par les effets sonores. Certains développent ainsi la musicalité de leur parole en conférant à leurs dires un tour esthétique entre chant et parole scandée. Outre l'attention pour le sens des discours tenus et I'agencement des identités citadines dont ils témoignent, I'analyse de cette parole scandée comme élément musical à part entière reste à faire, de même que celle de l'esthétique musicale spécifique du farah baladî contemporain.

\section{Jouer les salutations}

Les musiques de mariage sont passées au tamis de la saturation sonore, laquelle autorise toutes sortes d'approximations et d'hésitations dans la construction du paysage sonore par l'orchestre et le nabatshî, seul le silence étant proscrit. Les tempos sont accélérés pour permettre la danse, les chansons et les morceaux sont déformés par les contraintes spécifiques de la fête. L'improvisation et le jeu d'oreille sont la règle. La performance au cours du mariage baladî ${ }^{20}$ actualise un répertoire, reflète un style et s'organise autour d'interactions localisées sur la scène, la coulisse et la rue dans laquelle se tient le public.

Il existe pour ces accueils un motif musical intangible qui vient des traditions de fanfares (hasab Allah) et qui, même diversement exécuté, est toujours reconnaissable. II s'agit de " jouer les salutations » (idrâb al-salâm), lesquelles consistent en l'exécution d'une phrase musicale simple à l'accordéon ou à l'orgue, accompagnée par les percussions, afin de marquer l'accueil de la personne ${ }^{21}$. Des séquences rythmiques et mélodiques récurrentes contribuent à caractériser le mariage et, par extension, les musiques de danse. Le tît par exemple désigne un style consistant en une improvisation autour de quelques notes simples sur un tempo lent avec un jeu syncopé des percussions (construit sur des contretemps). Le tît se distingue de la tûba qui exclut les percussions et consiste en une improvisation modale de l'instrument mélodique (la plupart du temps sur les modes que les musiciens considèrent comme "populaires $»^{22}$ : ceux qui « marchent » comme le mode rast qui correspond à une gamme majeure avec les $3^{\mathrm{e}}$ et $7^{\mathrm{e}}$ degrés quart de ton et nahawand qui correspond à une gamme majeure avec les 3, 6 et $7^{\text {e }}$ degrés bémol).

Autre marqueur de la musique des afrâh, la surreprésentation des percussions locales, accompagnées d'une batterie, et qui comprennent généralement,

20. Pour une analyse plus large des musiques urbaines contemporaines en Égypte, voir Puig, 2006 4a.

21. Les notes sont: [CCCBCDCBAG/GABCDCBDC/GC], gamme de C (do) majeur. 22. Puig, 2006 4a. 
la tabla, tambour à fût unique avec membrane plastique, le $r^{\prime}$, tambourin sur cadre avec cymbalettes mécaniques et le duf, tambourin simple.

Enfin, les musiques de mariage de rue au Caire, et cela concerne l'ensemble des cérémonies de ce type, se caractérisent du point de vue des contenus par la variété des répertoires et des styles qui mêlent différentes formes d'improvisation musicale et rythmique destinées à la danseuse à des chansons aux statuts très différents, depuis la variété «savante » de l'âge d'or de la musique égyptienne jusqu'aux succès contemporains de la pop arabe. Toutefois, les musiciens de l'ancienne génération trouvent difficilement dans ces contextes de performance la possibilité de faire valoir une quelconque ambition artistique. Du moins s'en plaignent-ils et, de l'avis général, la fête de mariage est devenue, avant toutes choses, une affaire d'argent. Cela reste à discuter quand on observe la charge ludique de l'événement pour un public souvent fort nombreux. Le fait que les musiciens jouent pour des non musiciens, appelés à juger ou, à tout le moins, apprécier diversement leur art, a toujours été une source de tension au sein de la profession. J'ai d'ailleurs souvent été pris à témoin par mes informateurs qui nourrissent régulièrement de sourds ressentiments vis-à-vis d'un public considéré comme inculte musicalement, voire de façon générale (surtout quand il se trouve résider dans un quartier informel). Ainsi que le notait Howard Becker à propos des musiciens de danse de Chicago juste après la Seconde Guerre mondiale : " La relation d'opposition entre les musiciens et les non musiciens détermine non seulement la culture des musiciens, mais aussi le déroulement de leurs carrières » (1985, p. 127).

\section{CONCLUSION : LES HORIZONS URBAINS D'UNE ENQUÊTE}

Parler, filmer et enregistrer sont trois moments techniquement différents de l'enquête qui renvoient chacun à une dimension de la " performance sociale » que l'on peut considérer isolément. Tout d'abord, la façon dont les musiciens se constituent en groupe spécialisé doté de références communes fondées à la fois sur une pratique professionnelle et sur les lieux de cette pratique, une culture autant professionnelle qu'urbaine, ressort d'une démarche d'observation participante qui place la parole au centre du dispositif d'enquête et correspond à un moment fort de l'engagement personnel du chercheur. Les fêtes de mariage sont des situations privilégiées de la performance et constituent des moments d'orientation des regards, de mise en scène de la société citadine et de circulation des valeurs, des références et des codes constitutifs d'un ethos urbain. Elles sont filmées et analysées en priorité à partir de " carnets vidéos » ainsi constitués. Enfin, la matière musicale elle-même, dont les significations débordent le domaine musicologique pour recouvrir l'ensemble du paysage sonore, des pratiques musicales et des paroles chantées ou scandées, est isolée par l'enregistrement audio.

Derrière chacune de ces opérations, il y a un chercheur aux prises avec la présentation de lui-même et la négociation de son statut - il peut entendre non 
seulement ce qu'on pourrait avoir à lui dire, mais, également, ce qu'on pourrait lui faire écouter, et il ne faudrait pas non plus le « prendre pour une bille »! - qui doit apprendre sur le tas et dans l'urgence différentes techniques d'enregistrement audio et filmique, le tout en soutenant une discussion dans une langue étrangère avec plusieurs interlocuteurs. S'ajoutent à cela : la gestion, si possible pleine de tact, des multiples sollicitations qui ne manquent pas de se produire dans le commerce avec les informateurs, lorsqu'on tente de se retirer dignement d'une interminable veillée par exemple, les « pesanteurs » d'un terrain qui s'abîme dans les embouteillages du Caire lors de vaines tentatives de rassemblement des membres d'un orchestre disséminés dans la ville, mais également le subtil plaisir des sociabilités amicales auquel se superpose la satisfaction intellectuelle que revêt, parfois, I'activité sociale de recherche.

L'avantage de la grande ville est probablement qu'elle rend coutumière la rencontre entre personnes issues de milieux différents, banalisant un peu la présence de l'anthropologue dans la société considérée; après tout, lui aussi réside au Caire et y travaille éventuellement (ce qui permet de se prévaloir d'une diversité de rôles sociaux). Cette ethnologie discontinue sur un temps long est précieuse, car elle autorise la construction patiente de relations de terrain sans pour autant infliger aux informateurs une présence permanente trop envahissante ou, à l'inverse, des entretiens rapides effectués dans l'urgence d'un bref séjour, à même les tables des cafés, au gré de circonstances mal maîtrisées, destinés à nourrir ce que l'on pourrait qualifier « d'anthropologie des coins de table ».

Faisant partie de la ville, l'ethnographe ainsi conçoit bien comment le travail sur le milieu des musiciens est lié à l'exploration de la société citadine. Car il est difficile d'envisager la situation des musiciens sans se référer à la façon dont ils s'insèrent dans la société conventionnelle. Ils animent les fêtes familiales et pénètrent ainsi au cœur des quartiers au cours de leurs déplacements professionnels. Ils contribuent au chant de la ville comme à la vie sociale des citadins. Ils investissent des lieux variés et, en parallèle, sont maintenus dans une position de marginalité. Cette situation entre intimité urbaine et mise à distance sociale est liée à la caractéristique de la ville, « mosaïque de petits mondes juxtaposés, mais qui ne s'interpénètrent pas » (Park, cité par Hannerz, 1983, p. 318) et à la façon dont s'articulent sédentarité et mobilité. La relation entre proximité et distance qui caractérise la présence des musiciens dans la société citadine est en partie à l'origine d'un manque de reconnaissance sociale qu'ils partagent avec l'ensemble des professions mobiles qui heurtent les ordres locaux fondés sur la sédentarité (Puig, 2006 b).

Ces multiples prises sur les mondes de la ville offrent l'un des cadres d'intelligibilité et de mise en perspective des données, elles constituent l'horizon urbain de l'enquête. Un horizon fait de personnes et de relations, de significations et de mondes sociaux plus ou moins reliés à des territoires. En filigrane d'un terrain d'Égypte, cet ancrage dans la matérialité de la ville, ses ambiances et ses paysages sonores, permet ainsi l'exploration des multiples « voies urbaines » des musiques festives. 


\section{RÉFÉRENCES BIBLIOGRAPHIQUES}

Becker Howard, 1985, Outsiders, Étude de sociologie de la déviance, Métailié. Constant-Martin Denis, 2000, "Cherchez le peuple, Culture, populaire et politique », Critique internationale, $n^{\circ} 7$, p. 169-183.

Copans Jean, 2002, L'Enquête ethnologique de terrain, Paris, Nathan.

Depaule Jean-Charles, 1997, « Seigneur, prisonnier et poète », Communications, $n^{\circ} 65$, p. 21-34.

Doquet Anne, 1999, Les Masques dogons. Ethnologie savante et ethnologie autochtone, Paris, Karthala.

HANNERZ Ulf, 1992, Cultural complexity, studies in the social organization of meaning, Columbia University Press.

- 1983, Explorer - la ville, Paris, Les Éditions de Minuit.

Hughes Everett C., 1996, Le Regard sociologique, essais choisis, Paris, Éditions de l'École des Hautes Études en sciences sociales.

JOSEPH Isaac, 1997, "L'Hospitalité : Prises, réserves, épreuves », Communications, $\mathrm{n}^{\circ} 65$, p. 131-142.

LamBA Henri, 1911, Code administratif égyptien contenant: Les actes et lois organiques du Khédivat, les lois, décrets et règlements administratifs. Annotés de la jurisprudence mixte et indigène. Les lois financières, Paris, Librairie de la société du recueil Sirey.

MASquelier Bertrand et SiRAn jean-Louis (dirs), 2000, Pour une anthropologie de I'interaction, Rhétoriques du quotidien, Paris, L'Harmattan.

Puig Nicolas, 2001, "Le long siècle de I'avenue Muhammad Ali au Caire: d'un lieu et de ses publics musiciens », Égypte/Monde arabe, L'Égypte dans le siècle 1901-2000, $\mathrm{n}^{\circ}$ 4/5, p. 207-223.

- 2006 a, "Sha'abî," populaire " : usages et significations d'une notion ambiguë dans le monde de la musique en Égypte», Civilisations, Musiques "populaires»: catégorisations, circulations, enjeux, Sara la Menestrel (dir.), Université Libre de Bruxelles.

- 2006 b, «Amour, honte et prestige, Les musiciens de l'avenue Mohamed Ali entre intimité urbaine et mise à distance sociale », à paraître.

RABINOW Paul, 1988, Un ethnologue au Maroc : réflexions sur une enquête de terrain, Paris, Hachette.

Simmel Georg, "Essai sur la sociologie des sens» (trad. L. Gasparini) dans Sociologie et épistémologie, Paris, PUF, p. 223-238. 